\title{
Quantitative Ultrastructural Analysis of Hippocampal Excitatory Synapses
}

\author{
Thomas Schikorski and Charles F. Stevens \\ Molecular Neurobiology Laboratory and Howard Hughes Medical Institute, The Salk Institute, La Jolla, California 92037
}

From three-dimensional reconstructions of CA1 excitatory synapses in the rodent hippocampus and in culture, we have estimated statistical distributions of active zone and postsynaptic density (PSD) sizes (average area $\sim 0.04 \mu \mathrm{m}^{2}$ ), the number of active zones per bouton (usually one), the number of docked vesicles per active zone $(\sim 10)$, and the total number of vesicles per bouton ( 200), and we have determined relationships between these quantities, all of which vary from synapse to synapse but are highly correlated. These measurements have been related to synaptic physiology. In particular, we propose that the distribution of active zone areas can account for the distribution of synaptic release probabilities and that each active zone constitutes a release site as identified in the standard quantal theory attributable to Katz (1969).

Key words: synaptic vesicle; active zone; release; statistical distribution; hippocampus; release probability
Since the pioneering work of Katz and his collaborators on synaptic function (summarized in Katz, 1969) and that of the early electron microscopists on synaptic structure (Palay and Palade, 1955; Luse, 1956; Wyckoff and Young, 1956), a consistent goal of neurobiologists has been to identify the structural basis for the entities identified in Katz's theory of synaptic transmission (Katz, 1969). The synaptic vesicle is generally accepted as corresponding to Katz's quantum, although still without definitive evidence. The number of release sites $\left[N_{\mathrm{s}}\right.$ in Katz's (1969) scheme] associated with an axon has been identified with the total number of releasable vesicles, with the number of boutons, and with the number of active zones (Zucker, 1973; Jack et al., 1981; Korn et al., 1981; Neale et al., 1983; Redman and Walmsley, 1983; Walmsley et al., 1985; Pun et al., 1986; Propst and Ko, 1987; Redman, 1990; Walmsley, 1991; Pierce and Mendell, 1993; Pierce and Lewin, 1994). A possible anatomical counterpart of Katz's release probability $p$ has been subject to less speculation, but several authors have noted that $p$ might be related to synaptic size (see Pierce and Lewin, 1994).

With the development of optical techniques to study synaptic transmission (Betz and Bewick, 1992; Betz et al., 1992) and the extension of these techniques to hippocampal neurons in culture (Ryan et al., 1993; Ryan and Smith, 1995; Ryan et al., 1996), many synaptic properties can now be investigated in culture at the level of single central synapses. Although individual hippocampal synapses differ greatly from one another with respect to any one of these properties, a variety of the properties are highly correlated with the release probability of the synapse (Ryan et al., 1996; Murthy et al., 1997). To relate results from physiological investigations of synapse populations to synaptic structure, one must

\footnotetext{
Received March 25, 1997; revised May 5, 1997; accepted May 19, 1997.

This work was supported by the Howard Hughes Medical Institute, by National Institutes of Health Grant 5 RO1 NS 12961 (C.F.S.), and by Deutsche Forschungsgemeinschaft Grant Schi391/1-1 (T.S.). We thank C. Boyer and R. Jacobs for their technical assistance and Dr. T. Bartol, A. Shrom, and Dr. D. Wild for their help with the three-dimensional reconstructions.

Correspondence should be addressed to Dr. Charles F. Stevens, Molecular Neurobiology Laboratory and Howard Hughes Medical Institute, The Salk Institute, 10010 North Torrey Pines Road, La Jolla, CA 92037.

Copyright (C) 1997 Society for Neuroscience $0270-6474 / 97 / 175858-10 \$ 05.00 / 0$
}

know the statistical distribution of morphological characteristics. Current statistical information about hippocampal synapses comes from studies by Harris and Stevens (1989), Harris et al. (1992), Sorra and Harris (1993), and Harris and Sultan (1995), but this work has focused almost exclusively on the postsynaptic side, except for a reconstruction of nine representative boutons by Harris and Sultan (1995), and has not considered the structure of synapses in culture. One of the main goals of the research reported here is to determine the statistical distribution of presynaptic properties likely to be of physiological relevance for hippocampal synapses.

We have characterized rodent hippocampal excitatory synapses both in brain and in culture.

\section{MATERIALS AND METHODS}

\section{Terminology}

We use "active zone" in the same way as Couteaux (1961) does and reserve "release site" (sometimes used as a synonym for active zone) for the entity described in Katz's (1969) theory. "Morphologically docked vesicles" (or simply "docked vesicles") are those immediately adjacent to the active zone membrane; we recognize that these vesicles are a superset of the "biochemically" and "physiologically docked vesicles." "Synapse" (sometimes called "synaptic junction") is taken to be the unit of active zone, docked vesicles, and postsynaptic density (PSD); with this terminology a single bouton might make multiple synapses.

\section{Fixation and embedding}

Brain synapses. One adult mouse (strain C57 black 6) was perfused through the heart under deep Nembutal anesthesia $(80 \mu \mathrm{l} / 25 \mathrm{gm}$ body weight). A short perfusion with oxygenated saline [containing (in $\mathrm{mM}$ ) $\mathrm{NaCl} 120, \mathrm{KCl} 3.5, \mathrm{NaH}_{2} \mathrm{PO}_{4} 1.25, \mathrm{NaHCO}_{3} 26, \mathrm{MgCl}_{2} 1.3$, and glucose $10, \mathrm{pH} 7.2$ ] was followed by a perfusion with $4 \%$ glutaraldehyde in 100 $\mathrm{mM}$ phosphate buffer, $\mathrm{pH} 7.4$, at room temperature (RT). The brain was dissected and immersed in $4 \%$ glutaraldehyde in $100 \mathrm{~mm}$ phosphate buffer, $\mathrm{pH} 7.4$, at RT overnight. Vibratome sections $(300 \mu \mathrm{m})$ including the hippocampus were cut and post-fixed in $1 \% \mathrm{OsO}_{4}$ at $4^{\circ} \mathrm{C}$ for $1 \mathrm{hr}$. After dehydration in ethanol, the sections were contrasted en bloc in $0.5 \%$ uranyl acetate in $95 \%$ ethanol for $1 \mathrm{hr}$ and flat-embedded in Epon.

Culture synapses. Cells were plated at postnatal day 1 and grown for $14 \mathrm{~d}$ (for culture conditions, see Bekkers and Stevens, 1989). Rat hippocampal cells were immersed in $2 \%$ glutaraldehyde in $100 \mathrm{~mm}$ phosphate buffer at $4^{\circ} \mathrm{C}$ for $1 \mathrm{hr}$, washed in phosphate buffer, and post-fixed in $1 \% \mathrm{OsO}_{4}$ at $4^{\circ} \mathrm{C}$ for $1 \mathrm{hr}$. Cells were dehydrated in ethanol, contrasted en bloc in $0.5 \%$ uranyl acetate at RT for $50 \mathrm{~min}$, and embedded in Epon. 

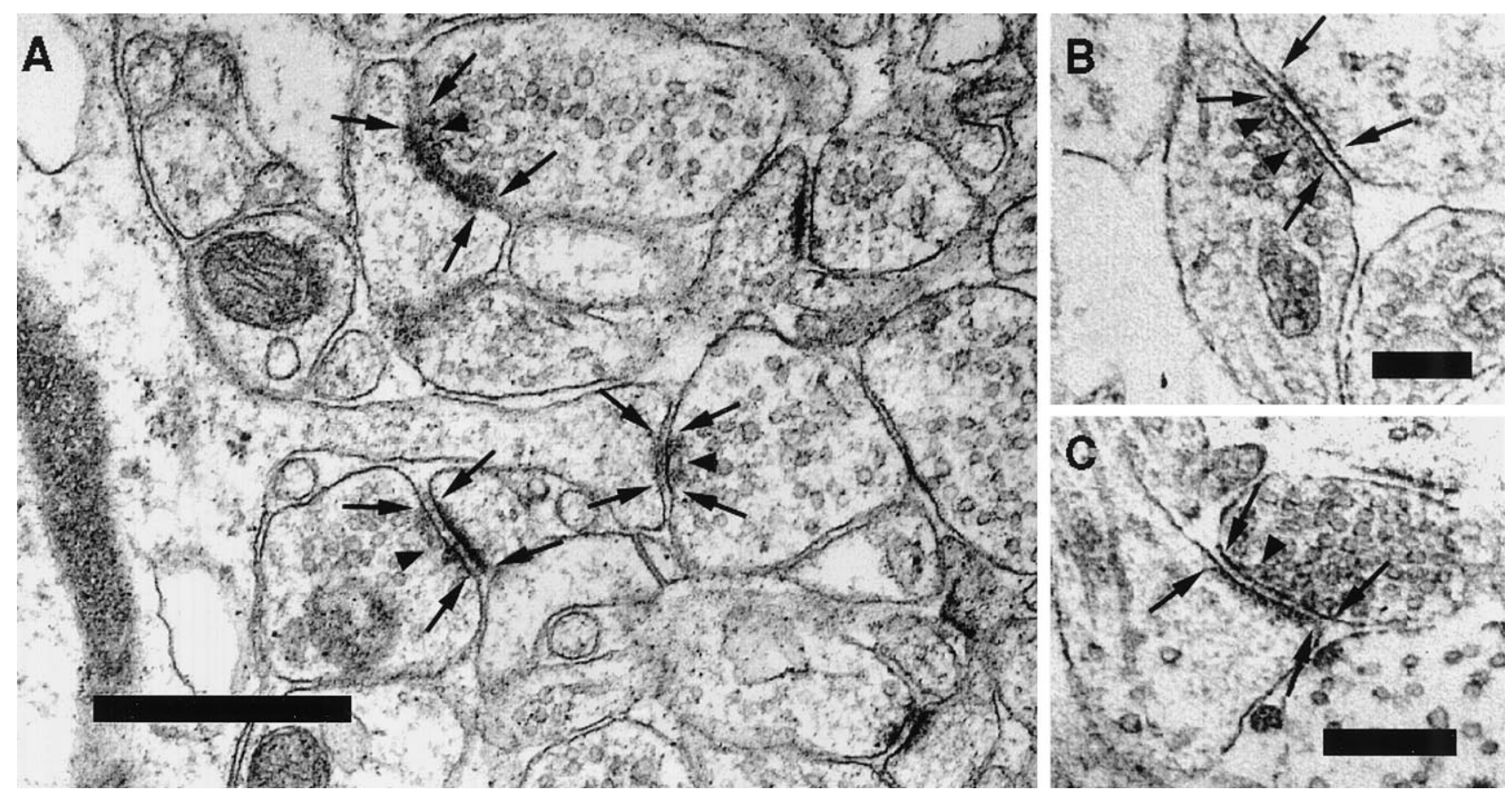

Figure 1. Sample electron micrographs used in our study. $A$, Electron micrograph depicting several synapses within the stratum radiatum in CA1 in the mouse hippocampus. The arrows indicate the borders of the active zones on the presynaptic side and of the postsynaptic densities on the spine. Examples of vesicles that are defined as docked in our study are marked by arrowheads. $B, C$, Electron micrograph showing the ultrastructure of two synapses from a hippocampal neuron grown in culture. Scale bars: $A, 0.5 \mu \mathrm{m} ; B, C, 0.25 \mu \mathrm{m}$.

Embedded vibratome sections were trimmed to blocks including the stratum (st.) pyramidale, the st. radiatum, and the st. lacunosum moleculare. Both specimens were cut serially at silver. Three series (magnification $14,000 \times)$ at different distances to the stratum pyramidale were photographed from 29 consecutive sections by using a Jeol 100 CX II electron microscope. For hippocampal neurons in cell culture, we photographed two series (magnification, 14,000×) from 20 serial sections cut at silver.

\section{Analysis}

Electron micrographs were scanned at 600 dpi resolution and analyzed by using the MetaMorph software (Universal Imaging Corporation, West Chester, PA). We analyzed 79 asymmetric contacts to spines of mouse hippocampal CA1 synapses; symmetric synapses or asymmetric shaft synapses were excluded from our measurements. All synapses within the selected area were included as long as the active zone was not cut tangentially as judged from the "smearing" of membranes. In the case of the hippocampal neurons in cell culture, we included 21 asymmetric contacts to spines and to dendritic shafts but excluded symmetric synapses from the analysis. Areas of active zones and of PSDs and volumes of boutons and of spine heads were measured by outlining the structure in consecutive sections using the Region Tools and the measurement function of the MetaMorph software. The final values were calculated by adding the product of the length or area times the section thickness $(60$ $\mathrm{nm}$ ) for all of the sections in which the structure appeared. A vesicle was counted as a docked vesicle when the vesicle membrane was immediately adjacent to the plasma membrane of the active zone (i.e., when a separation between the vesicular and plasma membrane was not resolvable). Vesicle diameters were calculated from the enface areas of vesicles photographed at high power $(72,000 \times)$. The diameter was taken as the diameter of a circle with the same area as the vesicle. Only vesicles that were contained completely within the 60 -nm-thick section were included.

Some very small active zones had a width of $\leq 60 \mathrm{~nm}$ and thus might appear in only a single section, and even large active zones that are traced across multiple sections were reconstructed in a discrete manner (see Fig. 5). The uncertainties in the active zone contures through a single section thickness give rise to errors in the area estimate. By examining upper and lower limiting sizes, we estimate that the error in any particular area could be as high as $20-30 \%$. We would overestimate the active zone area in some cases and underestimate it in others, so these errors would tend to cancel across a population of measurements for the larger active zones. For the smallest active zones, we probably systematically overestimated their area. However, errors for these zones should be $<50 \%$, because, e.g., an active zone that extended only halfway through a section thickness would have a $50 \%$ decrease in the electron density of the active zone membrane and we should have detected this.

Extracellular volume around a synapse was measured by outlining the area of the extracellular space. The outlines were contained within circles, decreasing appropriately in consecutive sections, that corresponded to a sphere with a radius of $0.5 \mu \mathrm{m}$ that centered on a particular reference synapse. We made no corrections for shrinkage during tissue preparation.

Several synapses were traced with Montage (written by R. Smith, University of Pennsylvania). Montage data were then rendered using the Nuages software (B. Geiger), and the volume rendered reconstructions were imported into AVS (Advanced Visual Systems, Inc.), in which the object properties (color, viewing angle, and transparency) were edited and printed.

\section{RESULTS}

Figure $1 A$ illustrates a typical region from a brain section with several synapses that were included in our study. The active zones and postsynaptic densities are defined by electron dense material close to the membrane. In addition, morphologically docked vesicles, a regular synaptic cleft, and alignment of presynaptic and postsynaptic structures mark the synaptic region. The active zones and PSDs are indicated by arrows. Figure $1, B$ and $C$, shows representative sections from a culture.

We reconstructed the boutons under study from 29 consecutive sections. An example of a reconstructed bouton is illustrated in Figure $2 A$ for the brain and in Figure $2 B$ for culture. In these illustrations the active zones and docked vesicles are indicated, but the nondocked vesicles are omitted for clarity.

We next consider briefly two features of synaptic structure that are important for interpreting physiological experiments: the 

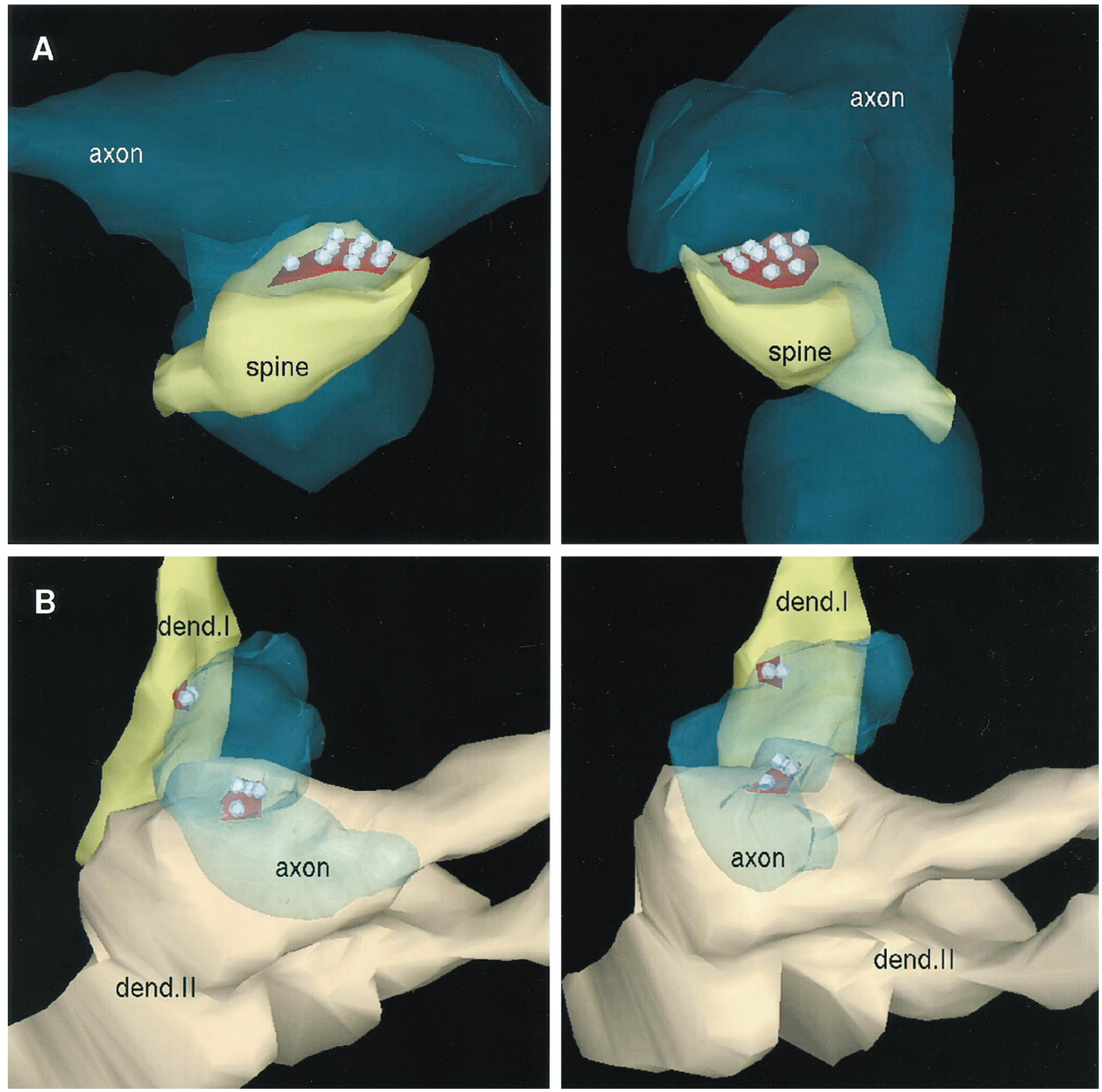

Figure 2. Three-dimensional reconstructions of synapses from serial sections. $A$, Two views of a brain synapse that is included in our study. The spine head (spine) is surrounded by two varicosities (axon). One of these varicosities forms a contact on the spine; the other is located beneath the spine. The right image presents a view from the right side of the structure in the left image. The bouton is transparent for the visualization of the active zone (red) and the nine docked vesicles ( gray). Nondocked vesicles are, for clarity, excluded. $B$, The reconstruction of two shaft synapses from cultured hippocampal neurons. Both synapses originate from the same varicosity (axon). The two different shades of yellow represent the two different dendritic shafts (dend. I, dend. II). Dend. II branches at the site of the synapse. The left and right images depict views of the same structure rotated by $\sim 30^{\circ}$. The red active zones and their docked vesicles are visible through the transparent axon; again nondocked vesicles are excluded for clarity.

distribution of the sizes of the synaptic vesicles and the diffusion volume for the neurotransmitter.

\section{Synaptic vesicle numbers and size distribution}

For brain synapses, the total number of vesicles per bouton averaged $270(\mathrm{SD}=176)$ with a range from 40 to 801 (for 17 boutons).

We measured the outer diameters of 343 synaptic vesicles that are (judged from an examination of serial sections) entirely contained within the 60 -nm-thick section. The outer diameters have a Gaussian distribution (Kolmogorov-Smirnov test, $p>0.2$ ) with a mean of $35.2 \mathrm{~nm}$, a SD of $3.4 \mathrm{~nm}$, and a coefficient of variation of 0.1 . The mean inner diameter is $23 \mathrm{~nm}$, calculated from the outer diameter by subtracting $6 \mathrm{~nm}$ for the thickness of the vesicular membrane.

\section{Neurotransmitter diffusion volume}

Because the width of the synaptic cleft in our material averaged $20.0(\mathrm{SD}=2.8) \mathrm{nm}$, the average volume of the synaptic cleft calculated from active zone size (see the next section for active 
zone and PSD areas) is $0.76 \times 10^{-3} \mu \mathrm{m}^{3}$ for the brain. This value, considered a mixing volume for the released neurotransmitter, is quite arbitrary, however, because it is calculated on the basis of active zone and PSD areas. The effective diffusion volume to which the neurotransmitter has rapid access could be very much larger. If one defines the "near" volume as the extracellular space within $0.5 \mu \mathrm{m}$ of the synapse (small molecules diff use $\sim 0.5$ $\mu \mathrm{m}$ in $0.25 \mathrm{msec}$ ), the diff usion volume for a single active zone in the brain was found to be $44 \times 10^{-3} \mu \mathrm{m}^{3}, \sim 50$ times the volume of the average synaptic cleft. We return to this issue of mixing volumes and their significance in Discussion.

\section{Brain active zones}

This section presents statistical data on the subcellular structure, the active zone, that is most intimately involved in neurotransmitter release.

Of the 71 brain boutons, $64(90 \%)$ exhibited only a single active zone, 6 had two active zones $(8 \%)$, and one had three active zones (2\%); multiple active zones at a single bouton never focused on the same postsynaptic spine. In culture, 11 of $16(69 \%)$ boutons possessed a single active zone, and five (31\%) had double active zones. These differences between brain and culture synapses are not statistically significant, but multiple active zones may actually be more common in culture than in brain.

In all cases, the active zone closely matches the PSD, as might be expected. Figure $3 A$ shows a plot of active zone area as a function of PSD area for 79 active zones in the brain; the correlation coefficient between these two variables is 0.97. A corresponding plot for culture synapses is presented in Figure $3 B$ in which the correlation coefficient is 0.974 (for 21 active zones).

The active zone area varies over a wide range (coefficient of variation $=0.56$ ), as does the number of docked vesicles (coefficient of variation $=0.54)$. The histogram of active zone areas (Fig. 4A) is broad with a mean of $0.039 \mu \mathrm{m}^{2}$ and an SD of 0.022 $\mu \mathrm{m}^{2}$; this histogram is satisfactorily fitted by a $\gamma$ density function (see Fig. 4 legend). If the average active zone were a circular disk, its diameter would be $0.22 \mu \mathrm{m}$.

Active zone area is linearly related to the volume of the bouton bearing the active zone. For 17 brain synapses that spanned the range of bouton volumes, we found that:

$$
V=1.47 A+0.018,
$$

where $V$ is the bouton volume $\left(\mu \mathrm{m}^{3}\right)$, and $A$ is the active zone area $\left(\mathrm{nm}^{2}\right)$; the correlation coefficient between $V$ and $A$ is 0.79 . The average bouton volume is $0.086 \mu \mathrm{m}^{3}$.

\section{Number of docked vesicles per active zone}

Like that of the active zone areas, the histogram of the number of docked vesicles per active zone (Fig. $4 B$ ) is broad with a positive skew and can also be fitted with a $\gamma$ density function (see Fig. 4 legend). The mean is 10.3 vesicles per active zone with a SD of 5.6. For our sample of 79 active zones, the minimum number of docked vesicles is two, and the maximum is 27 .

Although the active zone area and the number of docked vesicles per active zone both vary greatly from synapse to synapse, these two quantities are highly correlated as the scatter plot in Figure $4 C$ reveals. The correlation coefficient for brain active zone area and docked vesicles per active zone is 0.927 . The average active zone area per docked vesicle is $3.8 \times 10^{-3} \pm$ $0.93 \times 10^{-3} \mu \mathrm{m}^{2}$ (mean $\pm \mathrm{SD}$ ), which corresponds to one vesicle per $62 \times 62 \mathrm{~nm}$ square. The density with which docked vesicles occupy an active zone (the number of vesicles per area of active
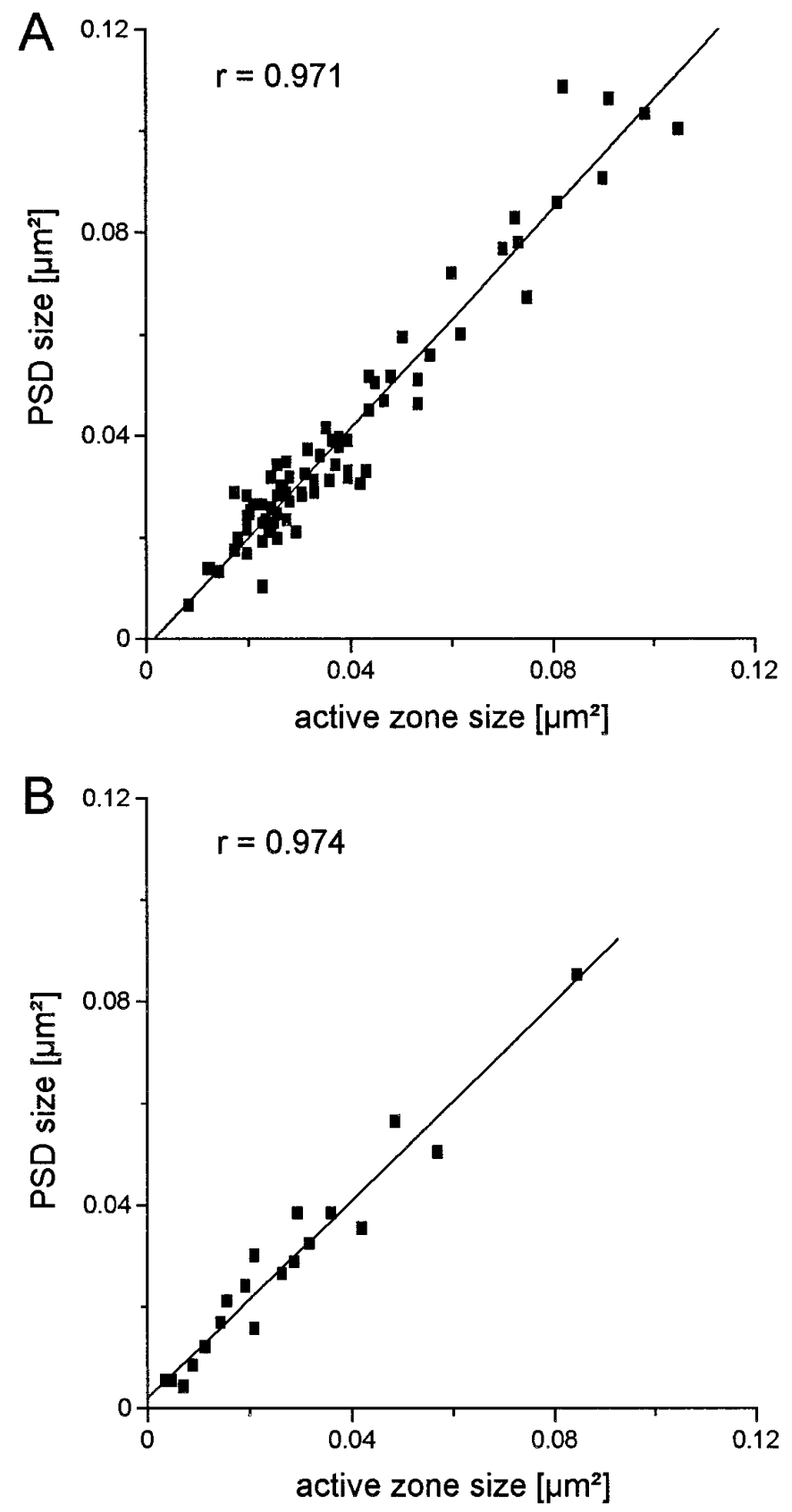

Figure 3. Correlation of active zone size with PSD size. A, Scatter plot comparing the sizes of 79 active zones from mouse brain with their corresponding PSD. The line indicates the linear regression with a correlation coefficient of 0.97. B, Same comparison of 21 active zones with PSDs from hippocampal neurons in culture. The correlation coefficient is 0.97 .

zone), relative to the maximal density observed, is normally distributed with a mean of 0.67 and a SD of 0.15 . Thus the active zones are, on average, filled to $67 \%$ of their observed maximal density.

\section{Active zone shapes}

Figure 5 illustrates 26 active zones with 157 docked vesicles (an average of 6 per active zone for this smaller sample) to indicate the range of active zone sizes and shapes encountered. In our sample, the largest active zone had a length of $0.8 \mu \mathrm{m}$ and a width of 0.26 $\mu \mathrm{m}$, whereas the smallest was 0.12 by $0.06 \mu \mathrm{m}$. The positions of 

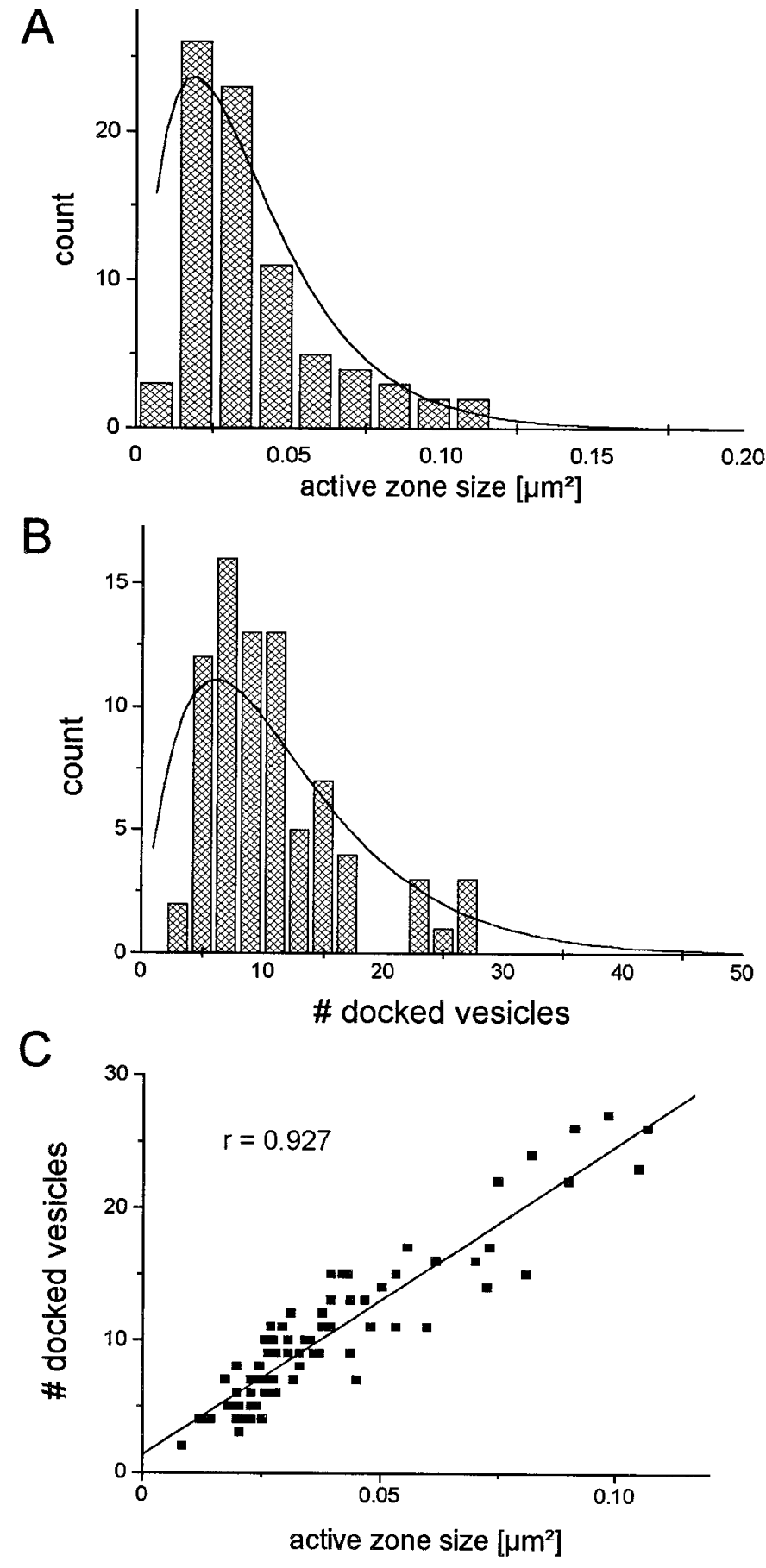

Figure 4. Comparison of active zone sizes with the number of docked vesicles for the brain synapses. $A$, Frequency distribution of active zone sizes for 79 active zones. The solid line is a $\gamma$ density function $(y=a \times$ $e^{-b x}$, with $a=3437$ and $\left.b=53\right)$. Mean $=0.039 \mu \mathrm{m}^{2} ; \mathrm{SD}=0.022 \mu \mathrm{m}^{2} . B$, Distribution of the number of docked vesicles for 79 active zones. The solid line is a $\gamma$ density function $\left(y=a \times e^{-b x}\right.$, with $a=4.98$ and $\left.b=0.165\right)$. Mean $=10.3$ vesicles per active zone; $\mathrm{SD}=5.6$. $C$, Scatter plot of active zone size and corresponding number of docked vesicles. The fitted linear regression is indicated as a solid line, and the correlation coefficient is 0.927 .

synaptic vesicles docked to the active zones are also illustrated in Figure 5. Note that the placement of the vesicles seems to be random, an appearance supported by the observation that the lateral intervesicle distances are approximately exponentially distributed with a characteristic length (measured from the edge of one vesicle to the edge of its neighbor) of $62.5 \mathrm{~nm}$. Compare this value with the overall density of one vesicle per $62 \mathrm{~nm}^{2}$.

Precisely how to identify a "functionally docked vesicle" in electron micrographs is not known. We have used the obvious definition above (close apposition of vesicle and plasma membranes for morphological docking), but we have also examined the effects of other definitions on our conclusions. When every vesicle within two vesicle diameters of the active zone is taken as docked, then the average number of docked vesicles per active zone increases from 10.3 to 18.8 (approximately twofold, as if vesicles are waiting in line to be released), whereas the correlation between the active zone area and the number of docked vesicles remains unaffected (correlation coefficient, 0.929). This positive correlation between the vesicle number and the active zone size persists even when we take as docked the total number of vesicles within the terminal (correlation coefficient, 0.79). Because the active zone area seems to predict the relative size of the docked pool when docked is defined in various ways, we shall prefer this measure for calculations that relate to the distribution of docked vesicles.

\section{Synapses in culture}

Because physiological experiments use hippocampal neurons both in slices and in culture, a comparison of synaptic structure in these two environments is important. In this section we compare boutons in culture with the data presented earlier for the brain. Note that our sample size for culture (16 boutons) is much smaller than that for the brain because of the much lower density of synapses in culture compared with that in the brain.

The average area of 21 active zones, reconstructed from 20 consecutive sections in culture, was $0.027 \mu \mathrm{m}^{2}$ with an SD of $0.019 \mu \mathrm{m}^{2}$. Our culture sample consisted of 14 active zones that were opposed to spines and 7 active zones that participated in shaft synapses; when multiple active zones were present, they did not oppose the same spine. The active zone areas are not significantly different from those obtained from the mouse brain. As we found in the brain, the culture active zone area is highly correlated with the PSD area (correlation coefficient $=0.974$; Fig. $3 B$ ) and with the number of docked vesicles; the average number of docked vesicles is 4.57 ( $\mathrm{SD}=3.00)$. The total number of vesicles per bouton averaged $195(\mathrm{SD}=154)$ with a range of 23 to 648 . Synapses in culture thus have fewer docked vesicles, although their active zone area is not significantly less than that for brain synapses.

Overall, the only statistically significant difference between the brain and culture synapses is the average number of docked vesicles per active zone (approximately half for culture). This difference cannot be attributed to the inclusion of shaft synapses in our sample from culture because the average number of docked vesicles was the same for both shaft and spine synapses in culture. Although not statistically significant, we note that more boutons in culture $(21 \%$, or 3 of 14 ) have multiple active zones than do boutons in brain $(11 \%$, or 7 of 64$)$.

\section{Summary of the quantitative presynaptic data}

Table 1 gives a summary of our data both for the mouse brain synapses and for the rat hippocampal neurons in culture.

\section{DISCUSSION}

The data reported here provide a picture of central synapses as rather variable structures with certain consistent characteristics. Most boutons possess only a single active zone that, as expected, 

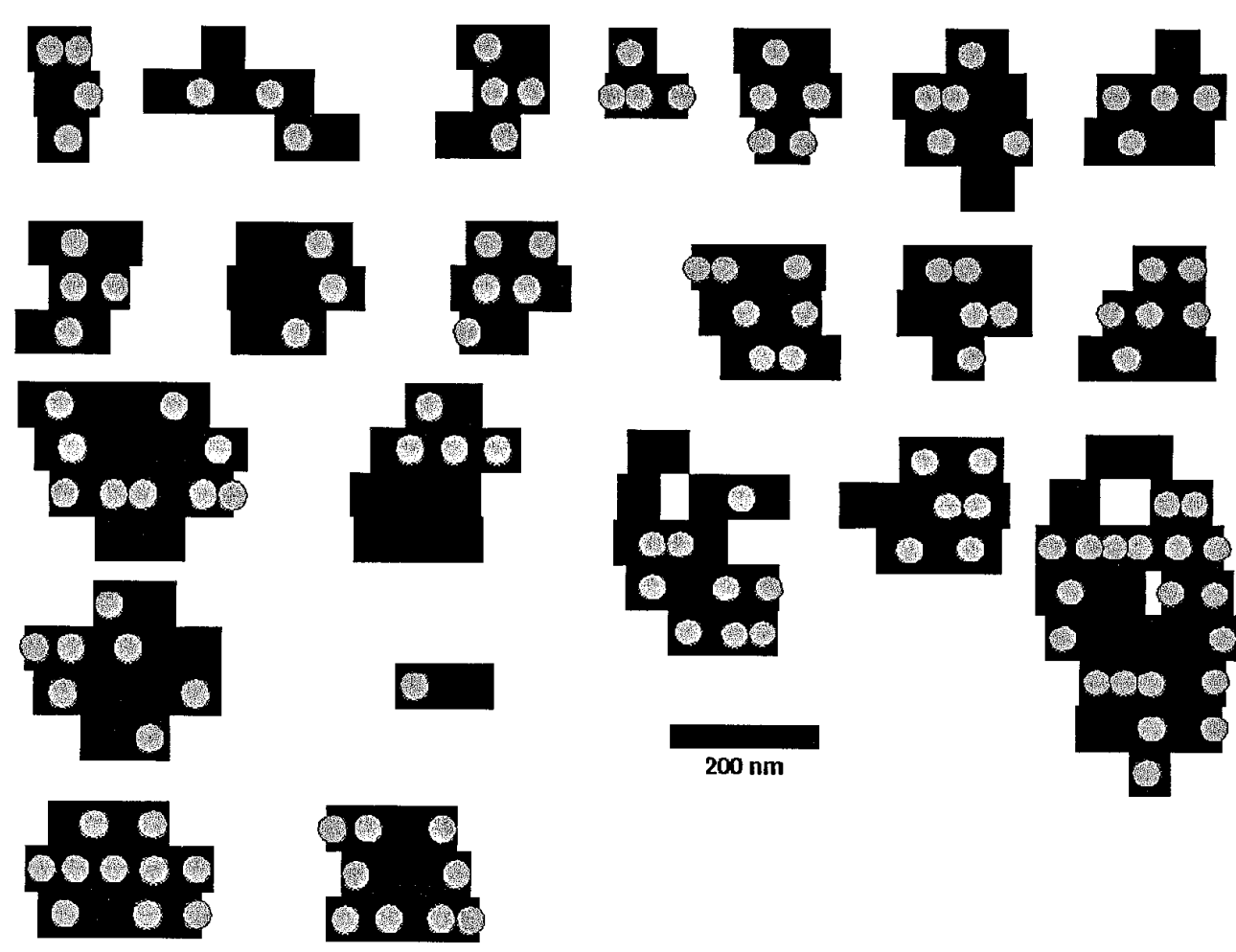

$200 \mathrm{~nm}$
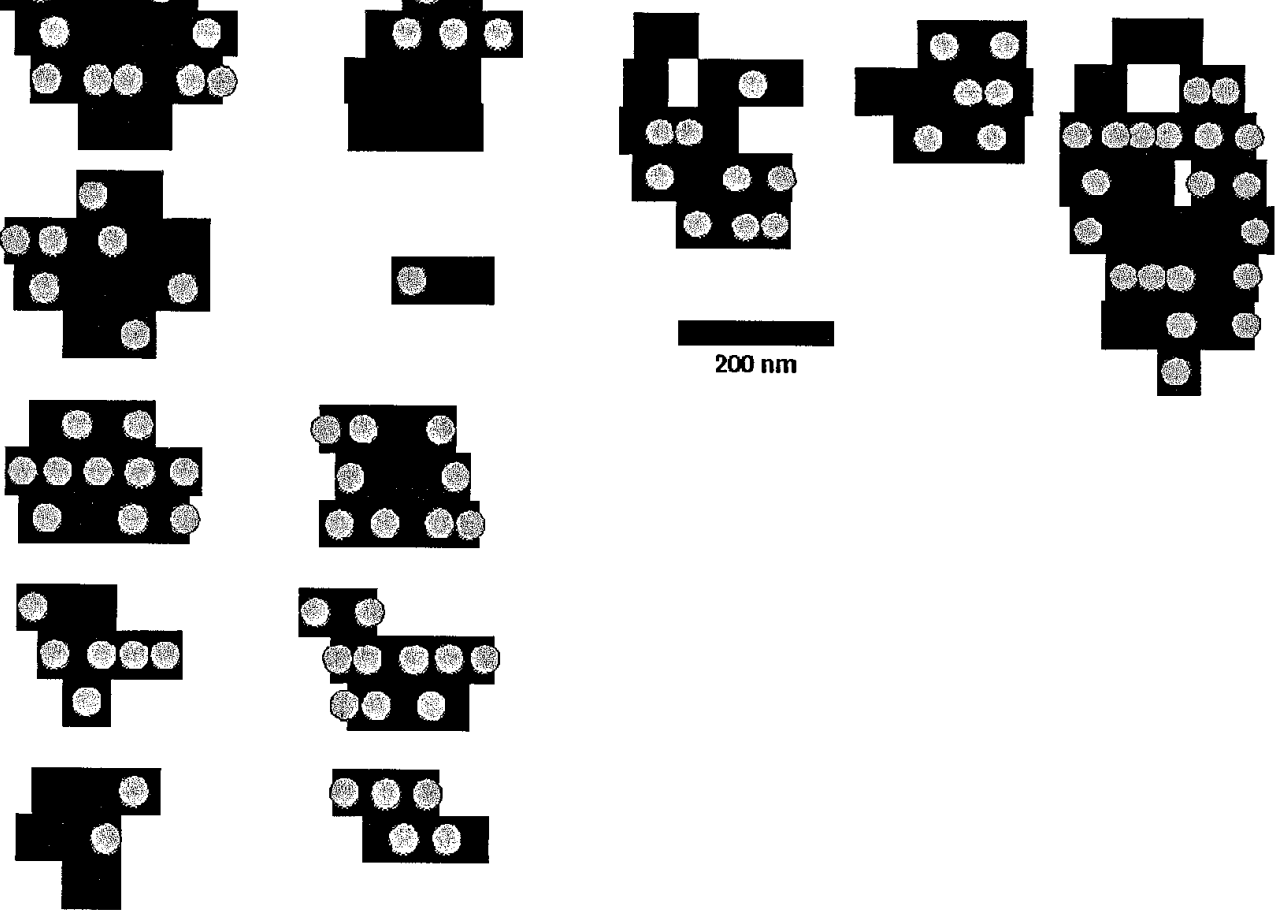

Figure 5. Depiction of 26 active zones, as viewed from within the bouton, reconstructed from serial sections. The black bars correspond to the active zone of a single section (thus the thickness of the bar equals $60 \mathrm{~nm}$ ), and the shaded circles indicate the position of docked vesicles. Note that because the section is approximately twice as thick as the vesicle diameter, the location of the vesicle within the section is unknown; all vesicles have been placed in the center of the section for illustration, but the actual vesicles would not be arranged in the neat rows as shown here. The point of representing the vesicles here is to indicate the extent to which areas of the active zone seem to be unoccupied. Many active zones could be described as round or oval, although distortions of these idealized forms are common. Two active zones were perforated. The occupancy of active zones by docked vesicles varies greatly.

is aligned quite precisely with the PSD. Furthermore, a tight relationship holds between the active zone size and the number of docked vesicles. But the size and shape of the active zone, the area of the PSD, the number of docked vesicles per active zone, and the size of the reserve pool of nondocked vesicles all vary greatly from synapse to synapse.

\section{Comparison with previous anatomical studies}

Although similar statistical studies of presynaptic structure have not, as far as we are aware, included cortical synapses, our observations are in good agreement with certain earlier findings for noncortical synapses. For frog cardiac autonomic synapses (Streichert and Sargent, 1989), turtle spinal cord synapses on motoneurons (Yeow and Peterson, 1991), and 1A synapses on mammalian spinal motoneurons (Pierce and Mendell, 1993), a linear relationship is reported between bouton volume and both the total number of synaptic vesicles and the active zone area. Also, spinal synapses exhibit a skewed distribution of active zone areas with a shape much like the one presented in Figure $4 A$, although the active zone areas of the spinal synapses are two and one-half (mammalian) to four (turtle) times those we find in hippocampus.

Several interesting differences are evident between the hippocampal boutons and others that have been characterized statistically. The great majority of hippocampal boutons have only a single active zone, whereas multiple active zones are common for spinal (an average of 6 active zones per bouton) (Yeow and Peterson, 1991; Pierce and Mendell, 1993), autonomic ( 1.5 active zones per bouton) (Streichert and Sargent, 1989), and thalamic ( $\sim 3-8$ active zones per bouton) (Hamos et al., 1987) synapses.

Another difference, bouton size, probably accounts for the occurrence of multiple active zones per bouton in these noncortical synapses and for the fact that hippocampal active zones are smaller than others. The volume of the hippocampal boutons is 10-100 times less than the volumes of the other boutons that have been characterized statistically, and all investigators find a linear 


\begin{tabular}{|c|c|c|}
\hline Presynaptic data & Brain & Cell culture \\
\hline Active zone & $0.039 \pm 0.022 \mu \mathrm{m}^{2}$ & $0.027 \pm 0.019 \mu \mathrm{m}^{2}$ \\
\hline PSD & $0.043 \pm 0.031 \mu \mathrm{m}^{2}$ & $0.028 \pm 0.020 \mu \mathrm{m}^{2}$ \\
\hline $\begin{array}{l}\text { Docked vesicle } \\
\text { number }\end{array}$ & $10.3 \pm 5.6$ & $4.6 \pm 3.0$ \\
\hline $\begin{array}{l}\text { Area per } \\
\text { docked vesicle }\end{array}$ & $62 \times 62 \mathrm{~nm}$ & $77 \times 77 \mathrm{~nm}$ \\
\hline Bouton volume & $0.086 \pm 0.049 \mu \mathrm{m}^{3}$ & $0.122 \pm 0.106 \mu \mathrm{m}^{3}$ \\
\hline Spine volume & $0.038 \pm 0.036 \mu \mathrm{m}^{3}$ & \\
\hline $\begin{array}{c}\text { Total number } \\
\text { of vesicles }\end{array}$ & $270 \pm 176$ & $195 \pm 154$ \\
\hline $\begin{array}{c}\text { Outer vesicle } \\
\text { diameter }\end{array}$ & $35.2 \pm 3.4 \mathrm{~nm}$ & \\
\hline
\end{tabular}

The left column summarizes values for mouse brain synapses as given in the text. The right column gives corresponding values for rat hippocampal neurons in culture; some entries do not appear in the text but are included here for completeness.

relationship between total active zone area and bouton volume. Because hippocampal synapses are small, their average active zone size is also small. As Yeow and Peterson (1991) first noted, active zones rarely exceed an area of $0.4 \mu \mathrm{m}^{2}$ (as if larger active zones will not function properly), so larger boutons seem to add more active zones to conform to the linear active zone area per bouton volume relationship without exceeding the upper limit for active zone size. As first reported by Hamos et al. (1987) and confirmed by the subsequent workers on various synapse types (Streichert and Sargent, 1989; Yeow and Peterson, 1991; Pierce and Mendell, 1993), the number of active zones is linearly related to bouton volume. Again, because hippocampal boutons are small, one would expect to find, as we do, only a single active zone per bouton if the linear active zone area per bouton volume relationship extends to cortical neurons. Although they did not perform a formal quantitative analysis, Peters et al. (1990) also note that the number of active zones per bouton correlates with bouton size in neocortex so that larger inhibitory boutons often exhibit multiple active zones whereas the smaller excitatory synapses generally possess only a single active zone, as we find for the hippocampus.

When specific comparisons can be made, our findings are generally in good agreement with previously published observations on hippocampal synapses. The most comparable study (Harris and Sultan, 1995) found, for the nine representative boutons they reconstructed, an average of 15.6 vesicles per active zone with a range of 2-36; this compares with our mean of 10.3 with a range of $2-27$ for the brain. The average bouton volume $(0.11$ $\mu \mathrm{m}^{3}$ ) reported by Harris and Stevens (1989) also agrees with our value $\left(0.086 \mu \mathrm{m}^{3}\right)$.

\section{Physiological implications}

\section{Cleft neurotransmitter concentration}

The concentration of agonist to which postsynaptic receptors are exposed is of physiological significance, and order of magnitude calculations are sometimes made by using the volume of a synaptic cleft and the number of transmitter molecules estimated to be contained within a single vesicle. For example, Harris and Sultan (1995) provide estimates from 0.24 to $11 \mathrm{~mm}$ for the peak glutamate concentration in the cleft using this procedure (the range results from variations in synaptic cleft size).

As noted earlier, taking the synaptic cleft volume as the appropriate one for calculating the peak glutamate concentration is rather arbitrary. An improved, but still quite approximate, method is to determine the effective mixing volume with the aid of the Einstein-Smoluchowski relationship (Einstein, 1926):

$$
x=\sqrt{2 D t}
$$

where $x$ is the root mean square distance $(\mu \mathrm{m})$ traveled in $t$ msec by a molecule with diffusion constant $D\left(\mu \mathrm{m}^{2} / \mathrm{msec}\right)$. A typical value for $D$, for a molecule the size of glutamate, would be $\sim 0.5$ $\mu \mathrm{m}^{2} / \mathrm{msec}$ for free diffusion (glutamate binding would slow diffusion but simultaneously lower concentrations). If one can estimate a characteristic mixing time, then the characteristic distance traveled in that time can be obtained from the Einstein-Smoluchowski relationship; and the accessible volume, that volume within a characteristic distance, can be determined from measuring from serial electron micrographs the extracellular space continuous with the synaptic cleft.

To estimate a characteristic time for this situation, one can consider the length of time required for glutamate to be released from its vesicle (Almers and Tse, 1990). For serotoninergic vesicles, the time constant for this release process is $\sim 0.25 \mathrm{msec}$ (Bruns and Jahn, 1995). The diffusion distance for a characteristic time of $0.25 \mathrm{msec}$ (assume a diffusion constant of $0.5 \times 10^{-5}$ $\mu \mathrm{m}^{2} / \mathrm{msec}$ ) would be $0.5 \mu \mathrm{m}$. The volume associated with this linear distance is $\sim 44 \times 10^{-3} \mu \mathrm{m}^{3}$, or $\sim 50$ times the average volume of a synaptic cleft, as noted earlier .

If the concentration of glutamate within a vesicle $(23 \mathrm{~nm}$ inner

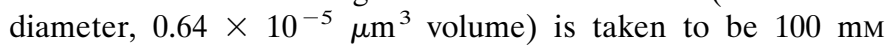
(Burger et al., 1989; Bruns and Jahn, 1995), the peak synaptic cleft concentration would be $\sim 0.6 \mathrm{~mm}$ for glutamate constrained to remain in the average synaptic cleft itself. The peak cleft concentration would be $12 \mu \mathrm{M}$ if the effective mixing volume is taken to be the contiguous space within $0.5 \mu \mathrm{m}$ of the synapse. This estimate $12 \mu \mathrm{M}$ is much lower than Harris and Sultan's (1995) value (an average of $2.5 \mathrm{~mm}$ for a $100 \mathrm{~mm}$ vesicle concentration) because they assumed a larger vesicle volume and took the mixing volume to be that of the synaptic cleft.

We have drawn three main conclusions about presynaptic structure that should relate to neurotransmitter release mechanisms.

\section{Active zone and release site correspondence}

First, we find that the majority of hippocampal boutons in the brain and in culture exhibit only a single active zone. Thus the study of single boutons, with minimal stimulation or optical methods, for example, reduces in effect to the study of single active zones. Because a single hippocampal bouton normally releases at most a single quantum of neurotransmitter (Stevens and Wang, 1994, 1995), Katz's (1969) $N_{\mathrm{s}}$, the number of release sites, would correspond to the number of active zones (and to the number of boutons). This conclusion is in agreement with earlier proposals (see Zucker, 1989; Redman, 1990; Korn and Faber, 1991; and Walmsley, 1991). Although an old idea dating from Zucker (1973), the release site and active zone identification has not been widely accepted. The main reasons for this lack of acceptance are uncertainties about the accuracy with which Katz's $N_{\mathrm{s}}$ could be determined by the quantal analysis used in the earlier studies. Several recent investigations (Walmsley, 1991; Pierce and Mendell, 1993; Rosenmund et al., 1993; Murthy et al., 1997) have confirmed earlier speculations that release probabilities for a single central axon vary considerably from site to site, and these studies find that most synapses have very low release 
probabilities. Under these circumstances, quantal analysis is very inaccurate for estimating $N_{\mathrm{s}}$ because the smallest sampling or systematic errors are disastrous for determinations of $N_{\mathrm{s}}$ because the Poisson limit is approached for a significant fraction of the synapses. The present identification of active zones with release sites is on firmer ground because quantal analysis was not required for counting release sites physiologically and because errors arising from the method of minimal stimulation would overestimate the quantal content as release probability changes (Stevens and Wang, 1994, 1995).

\section{Docked vesicle pool and releasable pool correspondence}

Second, the pool of morphologically docked vesicles averages $\sim 10$ and ranges from 2 to 27 in the brain (the range is 1-13 for cultured synapses), and we propose that these docked vesicles correspond approximately to the readily releasable pool determined physiologically, an idea put forward recently by Von Gersdorff et al. (1996) for a ribbon synapse. Although a direct test of this proposal is required before it can be accepted, the available data support the identification of the docked vesicles as the releasable pool (Südhof, 1995). The number of morphologically docked vesicles is in approximate agreement with the estimates of Stevens and Tsujimoto (1995), who found a releasable pool size that varied from 8 to 24 quanta for a small sample of cultured hippocampal synapses. L. Dobrunz and C. F. Stevens (unpublished observations) report an average pool size of $8.1 \pm 0.8$ for CA1 neurons in slices, in good agreement with the morphological estimates here.

The discrepancy between physiological (8-24) and morphological (1-13) estimates of the releasable pool size in culture could arise in at least two ways. First, the methods used by Stevens and Tsujimoto (1995) would tend to undercount boutons and therefore would overestimate the pool size; the physiological estimate might be too large. Second, the docked vesicle pool size determined morphologically might underestimate the actual size of the physiologically docked vesicle pool because of fixation artifacts; the morphological pool might be too small. Although one can never be certain about the relationship between electron micrograph images and the situation that holds for the living cell, we do not favor the fixation artifact alternative. Smith and Reese (1980) have reported that gluteraldehyde produces release at the frog neuromuscular junction, so that fixation might deplete the releasable pool. But we find that all neurotransmitter release is blocked with a time constant of $\sim 1 \mathrm{sec}$ in hippocampal cultures without significant "spontaneous" release produced by the fixative (C. Rosenmund and C. F. Stevens, unpublished observations). Release during fixation should have been detected because the postsynaptic glutamate receptors are fixed more slowly than is the release machinery; the postsynaptic responses to kainate decline approximately four times more slowly than does evoked release. We therefore have no evidence to suggest that the releasable pool is significantly depleted by fixation, although we cannot exclude this possibility.

\section{Docked vesicle pool and release probability correspondence}

Third, our determination of the distribution of active zone areas (and the number of docked vesicles) permits us to test the hypothesis that release probability is proportional to the number of docked vesicles (which is proportional to the active zone area). Murthy et al. (1997) have measured the distribution of release probabilities for a population of hippocampal boutons, and we can compare this distribution with the one obtained here if we make

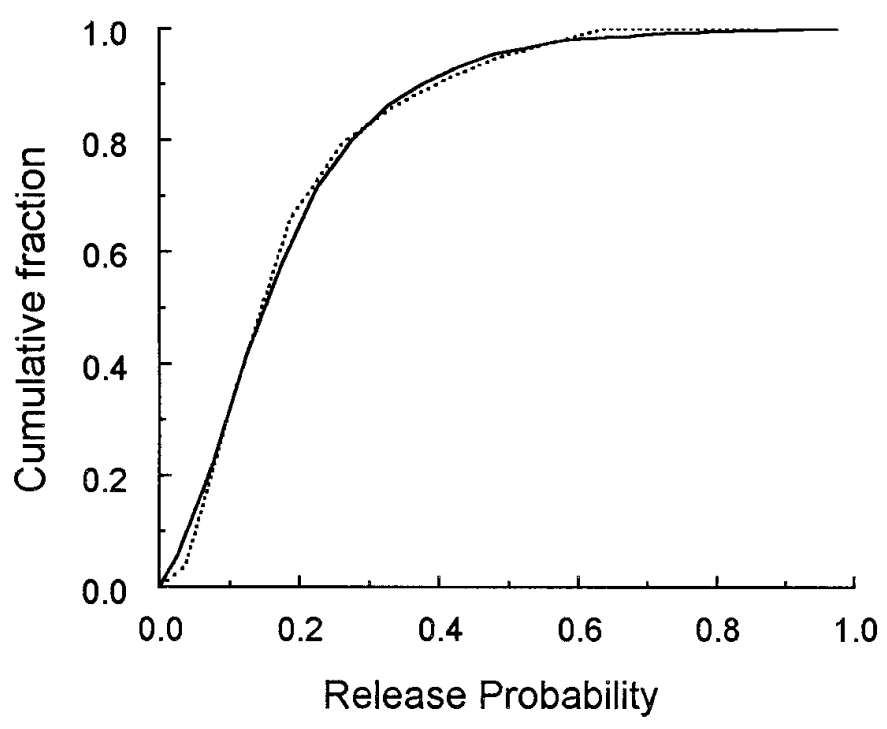

Figure 6. Comparison of the distribution of the active zone areas with the release probability. The solid line represents the release probability of hippocampal CA1 synapses as measured by Murthy et al. (1997). The dashed line gives the distribution of release probabilities predicted from the distribution of active zone areas (Fig. $4 A$ ) as described in Appendix. The theoretical and observed distributions are not significantly different (Kolmogorov-Smirnov test, $P>0.2$ ).

the simplest assumption of a proportionality between active zone area and release probability (see Appendix for a justification of this assumption). In Figure 6 we have plotted, in cumulative form, the experimentally derived release probability distribution (Murthy et al., 1997, their Fig. $2 B$ ) and the one predicted from the distribution of active zone areas (Fig. $4 A$ ). The two distributions (Fig. 6) are not significantly different (Kolmogorov-Smirnov test, $p>0.2$ ). A single scale factor relating active zone area to release probability was required for this match: active zone area $\left(\mu \mathrm{m}^{2}\right) \times$ 5.75 gives release probability. This value would correspond to a release probability of $\sim 0.03$ per docked vesicle.

Murthy et al. (1997) find that the lower release probability boutons exhibited the largest amount of paired pulse facilitation. This observation is in agreement with the finding of Bower and Haberly (1986) that a class of smaller boutons have greater facilitation. Although the relationship between the number of docked vesicles and release probability remains to be established at individual synapses, the data in Figure 6 indicate that the hypothesis is a tenable one.

\section{APPENDIX}

To compare our distribution of the active zone sizes (or the number of docked vesicles) with the physiological data on the distribution of release probabilities (see Fig. 6), we need a mathematical relationship that connects these two quantities. We made the simplest assumption: release probability is proportional to active zone area. The purpose of this Appendix is to justify this assumption. Rosenmund and Stevens (1996) proposed that, for the hippocampal synapses studied here, release probability is related to the number of docked vesicles; decreasing the pool of docked vesicles decreases release probability. Note that here "release probability" is defined as the probability that a bouton will release neurotransmitter and is, as will be seen below, distinct from the conditional probability that an individual vesicle will 
undergo an exocytotic event if no other vesicle does so (we call this the "exocytotic probability").

Stevens and Wang $(1994,1995)$ find that at most a single vesicle is released ordinarily by one bouton. This observation, together with the findings of Stevens and Tsujimoto (1995) and of Rosenmund and Stevens (1996) who reported that the rate of exocytosis is directly related to the releasable pool size, is consistent with the following simple mechanism: when the exocytotic probability is increased, the overall probability that transmitter will be released is, for short times, proportional to the number of vesicles available for release; but as soon as one of the vesicles is successful, this first exocytosis raises the energy barrier for other vesicles enough that a second exocytotic event hardly ever takes place. Before the first exocytotic event, then, release probability depends on all of the docked and cocked vesicles, but a winner-takeall mechanism limits release to at most one vesicle.

This view can be formalized as follows: We suppose that a nerve impulse has arrived at the terminal at time $t=0$. Let $f(t)$ be the probability that no exocytotic event has occurred up to time $t$, and call $\alpha(t)$ the individual vesicle exocytotic rate, the Poisson probability per second for each vesicle to undergo an exocytotic event. Each vesicle is assumed to be identical, and we have supposed that release occurs according to a Poisson process (Barrett and Stevens, 1972). The equation for the failure probability then is:

$$
d f(t) d t=-n \alpha(t) f(t),
$$

where $n$ is the number of vesicles in the readily releasable pool. The probabilistic exocytotic rate $\alpha(t)$ increases rapidly just after a nerve impulse arrives and then returns to low levels over several hundred microseconds. The solution to the above equation is:

$$
f(t)=e^{-n \int_{0}^{t} \alpha\left(t^{\prime}\right) d t^{\prime}} .
$$

We take the period of evoked release from $t=0$ to $t=T$ so that the probability that no release occurs in response to an arriving nerve impulse is $f(t)$. The value of the integral is, for a release period of fixed duration, just a constant $a$ :

$$
\int_{0}^{T} \alpha\left(t^{\prime}\right) d t^{\prime} \equiv a .
$$

The failure probability thus depends exponentially on the number of vesicles in the releasable pool

$$
f(T)=e^{-n a},
$$

and the release probability $p$ is:

$$
\begin{aligned}
p & =1-f(T) \\
& =1-e^{-n a} .
\end{aligned}
$$

For small values of $p$ this last relationship is approximately:

$$
p=n a,
$$

so release probability is proportional just to the releasable pool size. In this derivation we assumed that all vesicles had the same exocytotic probability and that they behaved independently up to the time that the winner-take-all mechanism operates. Both of these assumption can be relaxed, and the same exponential relationship between failure probability and pool size results. The value of $a$ might, under more complicated assumptions, differ from synapse to synapse and can change as the readily releasable pool is depleted.

\section{REFERENCES}

Almers W, Tse FW (1990) Transmitter release from synapses: does a preassembled fusion pore initiate exocytosis. Neuron 4:813-818.

Barrett EF, Stevens CF (1972) The kinetics of transmitter release at the frog neuro-muscular junction. J Physiol (Lond) 227:691-708.

Bekkers JM, Stevens CF (1989) NMDA and non-NMDA receptors are co-localized at individual excitatory synapses in cultured rat hippocampus. Nature 341:230-233.

Betz WJ, Bewick GS (1992) Optical analysis of synaptic vesicle recycling at the frog neuromuscular junction. Science 255:200-203.

Betz WJ, Mao F, Bewick GS (1992) Activity-dependent fluorescent staining and destaining of living vertebrate motor nerve terminals. J Neurosci 12:363-375.

Bower JM, Haberly LB (1986) Facilitating and nonfacilitating synapses on pyramidal cells: a correlation between physiology and morphology. Proc Natl Acad Sci USA 83:1115-1119.

Bruns D, Jahn R (1995) Real-time measurement of transmitter release from single synaptic vesicles. Nature 377:62-65.

Burger PM, Mehl E, Cameron PL, Maycox PR, Baumert M, Lottspeich F, DeCamilli P, Jahn R (1989) Synaptic vesicles immunoisolated from rat cerebral cortex contain high levels of glutamate. Neuron 3:715-720.

Couteaux R (1961) Principaux criteres morphologiques et cytochimiques utilisables aujourd'hui pour definir les divers types de synapses. Actual Neurophysiol 3:145-173.

Einstein A (1926) On the movement of small particles suspended in a stationary liquid demanded by the molecular-kinetic theory of heat. In: Investigations on the theory of the Brownian movement (Fürth R, ed). New York: Dover.

Hamos JE, Van Horn SC, Raczkowski D, Sherman SM (1987) Synaptic circuits involving an individual retinogeniculate axon in the cat. J Comp Neurol 259:165-192.

Harris KM, Stevens JK (1989) Dendritic spines of CA1 pyramidal cells in the rat hippocampus: serial electron microscopy with reference to their biophysical characteristics. J Neurosci 9:2982-2997.

Harris KM, Sultan P (1995) Variation in the number, location and size of synaptic vesicles provides an anatomical basis for the nonuniform probability of release at hippocampal CA1 synapses. Neuropharmacology 34:1387-1395.

Harris KM, Jensen FE, Tsao B (1992) Three-dimensional structure of dendritic spines and synapses in rat hippocampus (CA1) at postnatal day 15 and adult ages: implications for the maturation of synaptic physiology and long-term potentiation. J Neurosci 12:2685-2705.

Jack JJB, Redman SJ, Wong K (1981) The components of synaptic potentials evoked in cat spinal motoneurons by impulses in single group Ia afferents. J Physiol (Lond) 321:65-96.

Katz B (1969) The release of neural transmitter substances. Liverpool, England: Liverpool University.

Korn H, Faber DS (1991) Quantal analysis and synaptic efficacy in the CNS. Trends Neurosci 14:439-445.

Korn H, Triller A, Mallet A, Faber DS (1981) Fluctuating responses at a central synapse: $n$ binomial fit predicts number of stained presynaptic boutons. Science 213:898-901.

Luse SA (1956) Electron microscope observations of the central nervous system. J Biophys Biochem Cytol 2:531-542.

Murthy VN, Sejnowski TJ, Stevens CF (1997) Heterogenous release properties of visualized individual hippocampal synapses. Neuron 18:599-612.

Neale EA, Nelson PG, MacDonald RL, Christian CN, Bowers LM (1983) Synaptic interactions between mammalian central neurons in cell culture. III. Morphological correlates of quantal synaptic transmission. J Neurophysiol 49:1459-1468.

Palay SL, Palade GE (1955) The fine structure of neurons. J Biophys Biochem Cytol 1:69-88.

Peters A, Sethares C, Harriman KM (1990) Different kinds of axon terminals forming symmetric synapses with the cell bodies and initial axon segments of layer II/III pyramidal cells. II. Synaptic junctions. J Neurocytol 19:584-600.

Pierce JP, Lewin GR (1994) An ultrastructural size principle. Neuroscience 58:441-446.

Pierce JP, Mendell LM (1993) Quantitative ultrastructure of la boutons in the ventral horn: scaling and positional relationships. J Neurosci 13:4748-4763. 
Propst JW, Ko C-P (1987) Correlations between active zone ultrastructure and synaptic function studied with freeze-fracture of physiologically identified neuromuscular junctions. J Neurosci 7:3654-3664.

Pun RYK, Neale EA, Guthrie PB, Nelson PG (1986) Active and inactive central synapses in cell culture. J Neurophysiol 56:1242-1256.

Redman S (1990) Quantal analysis of synaptic potentials in neurons of the central nervous system. Physiol Rev 70:165-198.

Redman S, Walmsley B (1983) Amplitude fluctuations in synaptic potentials evoked in cat spinal motoneurones at identified group Ia synapses. J Physiol (Lond) 343:135-145.

Rosenmund C, Stevens CF (1996) Definition of the readily releasable pool of vesicles at hippocampal synapses. Neuron 16:1-20.

Rosenmund C, Clements JD, Westbrook GL (1993) Nonuniform probability of glutamate release at a hippocampal synapse. Science 262:754-757.

Ryan TA, Smith SJ (1995) Vesicle pool mobilization during action potential firing at hippocampal synapses. Neuron 14:983-989.

Ryan TA, Reuter H, Wendland B, Schweizer FE, Tsien RW, and Smith SJ (1993) The kinetics of synaptic vesicle recycling measured at single presynaptic boutons. Neuron 11:713-724.

Ryan TA, Ziv NE, Smith SJ (1996) Potentiation of evoked vesicle turnover at individually resolved synaptic boutons. Neuron 17:125-134.

Smith JE, Reese TS (1980) Use of aldehyde fixatives to determine the rate of synaptic transmitter release. J Exp Biol 89:19-29.

Sorra KE, Harris KM (1993) Occurrence and three-dimensional structure of multiple synapses between individual radiatum axons and their target pyramidal cells in hippocampal area CA1. J Neurosci 13:3736-3748.

Stevens CF, Tsujimoto T (1995) Estimates for the pool size of releasable quanta at a single central synapse and for the time required to refill the pool. Proc Natl Acad Sci USA 92:846-849.

Stevens CF, Wang Y (1994) Changes in reliability of synaptic function as a mechanism for plasticity. Nature 371:704-707.

Stevens CF, Wang Y (1995) Facilitation and depression at single central synapses. Neuron 14:795-802.

Streichert LC, Sargent PB (1989) Bouton ultrastructure and synaptic growth in a frog autonomic ganglion. J Comp Neurol 281:159-168.

Südhof TC (1995) The synaptic vesicle cycle: a cascade of proteinprotein interactions. Nature 375:645-653.

Von Gersdorff H, Vardi E, Matthews G, and Sterling P (1996) Evidence that vesicles on the synaptic ribbon of retinal bipolar neurons can be rapidly released. Neuron 116:1221-1227.

Walmsley B (1991) Central synaptic transmission: studies at the connection between primary afferent fibres and dorsal spinocerebellar tract (DSCT) neurones in Clarke's column of the spinal cord. Prog Neurobiol 36:391-423.

Walmsley B, Wieniawa-Narkiewicz E, Nicol MJ (1985) The ultrastructural basis for synaptic transmission between primary muscle afferents and neurons in Clarke's column of the cat. J Neurosci 5:2095-2106.

Wyckoff RWG, Young JZ (1956) The motoneuron surface. Proc R Soc Lond [Biol] 144:440-450.

Yeow MBL, Peterson EH (1991) Active zone organization and vesicle content scale with bouton size at a vertebrate central synapse. J Comp Neurol 307:475-486.

Zucker RS (1973) Changes in the statistics of transmitter release during facilitation. J Physiol (Lond) 229:787-810.

Zucker RS (1989) Short-term synaptic plasticity. Ann Rev Neurosci 12: 13-31. 